\title{
Counterfeit/copy drugs: patients safety in the heart
}

\section{Y. Capan}

\author{
Hacettepe University, Faculty of Pharmacy, Department of Pharmaceutical Technology, Ankara, \\ Turkey
}

\begin{abstract}
Some studies demonstrate that a large proportion of copy drugs contain lower levels of active substances and higher impurity levels than reference drugs. The number of copy drugs failing to meet internationally recognised quality criteria consequences of patients receiving lower doses of active substances than expected. In addition, possible clinical effect of impurities must be considered. Counterfeiting of drugs exhibits a significant and growing threat to human health and public safety. It also results an economic problem for innovator drug manufacturers, undermining their revenues and reputation. Moreover, it negatively affects the confidence of the public in their medicines and the credibility of national health and enforcement authorities. Although precise and detailed data on counterfeit and substandard drugs are impossible to obtain, it is widely assumed that $10 \%$ of medicines worldwide are likely to be counterfeit while it is estimated that $5 \%$ of all world trade in branded goods is counterfeit, leading to huge financial losses for the pharmaceutical industry. But much more important from public health point of view, is that such products may lead to a great health risk. The essence of counterfeit products and the reason they are so dangerous is the complete absence of quality control, since they are often indistinguishable from the genuine product. Internet-sourced drugs are often considered suspect. The World Health Organization (WHO) reports that drugs from websites that conceal their physical address are counterfeit in over 50 percent of cases; the U.S. Food and Drug Administration (FDA) works with the National Association of Boards of Pharmacy (NABP) to regulary update a list of websites likely to sell drugs that are illegal or of questionable quality genuine drugs.
\end{abstract}

Correspondence: Y. Capan, Hacettepe University, Faculty of Pharmacy, Department of Pharmaceutical Technology, Ankara, Turkey.

Key words: counterfeit drugs, copy drugs, internet-sourced drugs, genuine drugs.

(C) Copyright Y. Capan, 2011

Licensee PAGEPress, Italy

Thalassemia Reports 2011; 1(s2):e28

doi:10.4081/thal.2011.s2.e28

This article is distributed under the terms of the Creative Commons Attribution Noncommercial License (by-nc 3.0) which permits any noncommercial use, distribution, and reproduction in any medium, provided the original author(s) and source are credited.

Parts of this work were presented at the "12th International Conference on Thalassemia and Hemoglobinopathies”, Antalya (Turkey), 11-14 May 2011. 